\title{
Métodos de Pronóstico por Indicadores dentro de la Gestión del Conocimiento Organizacional
}

\author{
Gustavo Illescas ${ }^{1}$, María Isabel Sanchez-Segura ${ }^{2}$, Graciela Ana Canziani ${ }^{3}$ \\ illescas@exa.unicen.edu.ar, misanche@inf.uc3m.es, canziani@exa.unicen.edu.ar \\ ${ }^{1}$ Departamento de Computación y Sistemas, Facultad de Ciencias Exactas, Universidad Nacional del Centro \\ de la Provincia de Buenos Aires (UNCPBA), Tandil, Argentina \\ ${ }_{2}^{2}$ Departamento de Informática de la Universidad Carlos III (UC3M) - Madrid, España \\ ${ }^{3}$ Departamento de Matemáticas, Facultad de Ciencias Exactas, Universidad Nacional del Centro de la \\ Provincia de Buenos Aires (UNCPBA), Tandil, Argentina
}

DOI: 10.17013/risti.e3.29-41

Resumen: Frecuentemente se manifiestan dificultades en la toma de decisiones debido a que no se cuenta con mecanismos robustos que permitan obtener indicadores predictivos utilizando como base los datos del pasado. Resulta entonces de vital importancia contar con herramientas y procedimientos que permitan aplicar métodos de pronósticos de indicadores para comprobar su factibilidad y uso dentro de la gestión del conocimiento organizacional. Esto ha motivado nuestra investigación en pos de un marco metodológico que permita la ponderación de la evolución de los indicadores. El presente trabajo propone un aporte científico en el campo de la mejora de la gestión por indicadores, en organizaciones conscientes de la importancia de tomar decisiones estratégicas a partir de la evolución de los indicadores que marcan el rumbo y el estado de salud de la organización.

Palabras-clave: Indicadores; Gestión del conocimiento; Pronóstico.

\section{Forecasting methods by indicators within the management of organizational knowledge}

\begin{abstract}
Often difficulties in decision-making arise because of the lack of robust mechanisms permitting to obtain predictive indicators based on past data. Hence, it is vitally important to have tools and procedures that allow us to apply forecasting methods by indicators to corroborate its feasibility and use within the management of organizational knowledge. This has motivated our research for a methodological framework that may allow weighting the indicators' evolution. This work proposes a scientific approach in the field of improvement of management by indicators in organizations that are conscious about the importance of strategic decision-making based on the evolution of indicators that mark the direction and health status of the organization.
\end{abstract}

Keywords: Indicators; Knowledge Management; Forecast. 


\section{Introducción}

La necesidad manifestada como "más frecuente" para el trabajo con indicadores es, sin lugar a dudas, lograr medir, o reflejar mediante un valor, el estado de una cuestión de interés expresado por una variable.

Más allá de la definición de qué es un indicador (un índice, un coeficiente, un dato simple), para ejemplificarlos utilizaremos elementos a los que estamos acostumbrados ya que en nuestra vida cotidiana estamos rodeados de ellos. Por este motivo hemos tomado la imagen de un termómetro, es algo concreto, un objeto destinado a medir. El mismo muestra visiblemente el resultado de un proceso de medición que traduce la temperatura del ambiente o de un cuerpo a un valor y una unidad. Tenemos por un lado un elemento que es quien toma la medición, un objeto que es sobre el que se toma la medición, un valor que entrega el resultado de la medición y una serie de parámetros sobre las que se toma una determinada acción o decisión.

Los valores se encuentran distribuidos a lo largo de una escala con un máximo y un mínimo que indican los extremos que puede alcanzar. Para mostrar el valor actual, este tipo de representación completa el objeto elegido (en este caso un tubo) con un color; para una mejor interpretación se han colocado otros elementos que muestran rápidamente que tan cerca estamos de un extremo u otro y que significan (mayor frío/ calor).

Toda medición implica que existió antes un interés por realizar un control para luego establecer una acción. Siguiendo el ejemplo del termómetro, un caso evidente lo hemos percibido en la temperatura corporal (la fiebre); en salud por lo general se habla de una temperatura normal $\left(36^{\circ}\right)$, una de "afiebramiento" $\left(38^{\circ}\right)$ y una temperatura máxima $\left(40^{\circ}\right)$ que nos señala un estado más que delicado. Todas las situaciones requieren algún grado de atención, para la temperatura normal todo indica que nos encontramos bien, para la de afiebramiento ya implica actuar rápidamente (por ejemplo con una medicación antitérmica) y para la máxima, dado la gravedad (riesgo de convulsión) la necesidad de atención es urgente.

Al hablar de indicadores se introduce el concepto de alarma y aquí se vuelve a lo cotidiano ya que el elemento mayormente utilizado es el semáforo. El mismo semáforo es un indicador de una acción a tomar de acuerdo al estado en que se encuentra. Así por ejemplo, si el valor de la temperatura se encuentra "dentro de un determinado rango" ACEPTABLE, se la visualiza en color AMARILLO, si estuviera dentro de los valores deseables u ÓPTIMOS en VERDE y si estos fueran CRÍTICOS o inaceptables, en ROJO. Estos estados y sus puntos de corte o umbrales (entre que valores se encuentra por ejemplo el estado ACEPTABLE), los define una persona basado en su experiencia.

Una revisión exhaustiva sobre el tema gestión por indicadores nos deja como conclusión que, a partir de la consolidación y éxito del modelo propuesto por Kaplan y Norton (1996), tanto ellos como otros autores sólo han avanzado en la aplicación del Cuadro de Mando Integral (CMI) en casos de estudio. Así entonces encontramos numerosos casos de éxito en distintas organizaciones: pequeñas y medianas industrias (PYMES), municipios, universidades, agroindustrias, turismo (más específicos aún en hotelería). 
En síntesis, ellos aplicaron la metodología propuesta y obtuvieron el éxito prometido por esto pero no agregaron valor al método. De esta manera siguen sin resolverse las cuestiones que fueron pasadas por alto, algunas de las cuales han motivado esta investigación.

$\mathrm{Al}$ respecto existen experiencias sobre casos puntuales como en la utilización de CMI y cadenas de Markov para la toma de decisiones sobre nuevos productos (Chan \& Ip, 2010); la simulación de indicadores de CMI soportados por cadenas de Markov Montecarlo (Köppen et al. 2007); la validación de las relaciones causa-efecto a través de redes de Bayes (Blumenberg et al. 2006). A pesar de los aportes que estos trabajos realizan, no concluyen sobre las dificultades que encierran en sí misma la gestión por indicadores (Illescas et al. 2014) entre ellas la falta de mecanismos de validación y la ausencia de integración de los métodos probados como parte de las metodologías (por ejemplo al CMI) por lo que muestran más bien casos exitosos aislados.

La gestión por indicadores está presente en multitud de procesos de toma de decisiones en la industria, desde la gestión operacional pasando por las decisiones directivas, hasta completar la estrategia para asegurar el éxito de la organización. Desde nuestra perspectiva, la gestión por indicadores se compone no solo de encontrar un conjunto de estos que son de interés para el monitoreo de la organización (como lo trata la mayoría de la bibliografía referenciada), sino también de un conjunto de reglas para el mantenimiento y actualización de los registros históricos, como así también del uso que se les da a los mismos y las responsabilidades que se les asigna a los usuarios de estos indicadores.

Frecuentemente se utiliza la denominación de "Indicadores de gestión” como una clasificación entre tantas, referido a cuáles son los indicadores que se consideran más importantes o necesarios para la gestión de una organización. En este trabajo nos referimos al concepto de "Gestión por indicadores" que se encuentra relacionado a cómo administramos nuestra organización mediante el uso de indicadores de cualquier categoría que se trate, de manera tal que eviten caer en un modelo estático de simple observación de cómo ocurren las cosas (Illescas et. al. 2013).

Visto el contexto de la gestión por indicadores, la importancia de la misma y la dificultad que existe actualmente para evaluar métodos que faciliten el análisis y pronóstico de indicadores, se tiene aquí como objetivo presentar las herramientas y los procedimientos que permitan aplicar métodos para comprobar su factibilidad y uso dentro de la gestión del conocimiento organizacional.

\section{Aplicación de métodos matemáticos}

Esta sección está destinada a mostrar la aplicación de métodos matemáticos para el análisis y pronóstico de indicadores. Los métodos matemáticos utilizados han sido empleados con éxito en numerosas aplicaciones incluso en indicadores pero a la manera tradicional, la perspectiva con la que se los utiliza en este trabajo es innovadora y proporciona un valor agregado al análisis de los datos para la gestión de la organización. Para la aplicación sistemática de los métodos seleccionados hemos diseñado un conjunto 
de procedimientos, como así también planteamos la forma en que se interpretan los resultados para concluir apropiadamente.

Para mostrar los resultados de nuestra investigación hemos elegido un caso de aplicación dentro del contexto de las finanzas referido a la determinación de la morosidad de una cartera de clientes (proyecto de cooperación con una empresa de electrodomésticos). Para abordar el caso se propuso una serie de indicadores de gestión entre los que se encuentran:

- Crédito total en monto: cantidad de dinero que actualmente adeudan el total de los clientes

- Cantidad total de morosos: aquellos clientes que posean cuotas impagas

- Cantidad de clientes incobrables: quienes adeuden cuotas por más de una determinada cantidad de tiempo

- Porcentaje de clientes incobrables:

(Cantidad de cliente incobrables / Cantidad total de morosos) ${ }^{*} 100$

Las tablas de datos, presentadas más adelante, contienen la aplicación del caso de estudio citado anteriormente, donde los valores mostrados están expresados en pesos (\$) y han sido pre-procesados para llevarlos a valor actual tomando como criterio en índice de inflación establecido por el Banco Nación argentino.

En particular, el ejemplo se ha acotado a mostrar resultados del indicador "Crédito total en monto" que resume información sobre la cantidad de dinero otorgada en crédito por compras a los clientes de la organización. El registro de este indicador se realiza mensualmente como una sumatoria total de las cuotas adeudadas por la totalidad de los clientes. En particular, cada sucursal tiene el registro propio del crédito y luego la central lo sumariza para obtener el monto global.

El hecho de haber documentado los procedimientos nos garantiza poder aplicarlos de igual forma en el estudio de otros casos.

\subsection{Proceso de análisis de indicadores}

El procedimiento de análisis de los indicadores consta de una serie de eventos que se muestran en la tabla 1, los cuales son implementados en un prototipo de software diseñado a tal fin, el cual brinda la posibilidad de incorporar indicadores a una Base de Datos (BD) independientemente del contexto de aplicación. El analista procede a la selección de un indicador de su interés para aplicar uno de los métodos matemáticos lo que requiere también la elección del período de análisis. El proceso de clasificar los indicadores dejará como resultado los rangos de criticidad para el período y la evaluación de cada valor del indicador para establecer su estado. A partir de allí el siguiente proceso permite pronosticar sobre el valor y el estado del indicador. Para asegurar el correcto desarrollo de las herramientas matemáticas, los resultados se validan realizando un procedimiento similar pero esta vez en herramientas externas, esto es, a partir de software reconocido (ver sección 2.2). Una vez validados los resultados, esta etapa no formará parte del proceso general hasta que se implemente una nueva técnica. 
Tabla 1 - Proceso de análisis de indicadores

\begin{tabular}{lll}
\hline Evento & Descripción & Tipo \\
\hline $\begin{array}{l}\text { Seleccionar } \\
\text { indicadores }\end{array}$ & $\begin{array}{l}\text { Dentro de los indicadores que el decisor puede analizar, según } \\
\text { la configuración establecida. En caso de que se encuentre } \\
\text { desactualizado, este notificará previo al análisis. }\end{array}$ & $\begin{array}{l}\text { Datos / } \\
\text { Sistema }\end{array}$ \\
\hline $\begin{array}{l}\text { Elegir el período } \\
\text { de análisis }\end{array}$ & $\begin{array}{l}\text { Incluye el agrupamiento que se desee realizar para el análisis, por } \\
\text { ejemplo los datos de los últimos 2o años tomados de a 5. }\end{array}$ & $\begin{array}{c}\text { Datos / } \\
\text { Sistema }\end{array}$ \\
\hline $\begin{array}{l}\text { Clasificar } \\
\text { indicadores }\end{array}$ & $\begin{array}{l}\text { Provee un método para clasificar el indicador y poder así determinar } \\
\text { los estados observables junto a los umbrales de las alarmas. A partir } \\
\text { de allí cada valor del período será clasificado con el estado que } \\
\text { determinan los umbrales. }\end{array}$ & $\begin{array}{l}\text { Proceso } \\
\text { Sistema }\end{array}$ \\
\hline $\begin{array}{l}\text { Aplicar método } \\
\text { de pronóstico }\end{array}$ & $\begin{array}{l}\text { Los diversos métodos implementados serán aplicados para } \\
\text { pronosticar tanto valores como así también estados futuros del } \\
\text { indicador. }\end{array}$ & $\begin{array}{l}\text { Proceso } \\
\text { Sistema }\end{array}$ \\
\hline $\begin{array}{l}\text { Validar resultado } \\
\text { Durante el desarrollo de los métodos matemáticos, se realiza }\end{array}$ & $\begin{array}{l}\text { Proceso } \\
\text { la validación de los resultados comparándolos con aplicativos } \\
\text { existentes. }\end{array}$ & Sistema \\
\hline
\end{tabular}

\subsection{Métodos para la clasificación y pronóstico de indicadores}

Respecto de los métodos matemáticos los hemos pre-seleccionado a partir del uso en diferentes casos de estudio realizados en tesis de grado y en cátedras (en la carrera de Ingeniería de Sistemas) para verificar su adaptabilidad al caso de indicadores ${ }^{1}$. El universo de métodos de partida fue tomado de la estadística tradicional, regresión lineal, lógica difusa, clustering y multicriterio.

Así trabajamos con dos propiedades delos indicadores: valory estado. Cuando trabajamos sobre los estados, reducimos nuestro análisis a los tres posibles de la semaforización. De acuerdo con las propiedades elegidas precedentemente, las condiciones previas para el análisis con métodos matemáticos son las siguientes:

- Fecha de registro del valor del indicador: para la utilización de ciertos métodos es importante a la hora de seleccionar los subconjuntos de datos para el análisis contar con un volumen tal que permita ser aplicado. También puede ser de utilidad conocer previamente la variabilidad de los datos.

- Valor del indicador: es de interés detectar datos anómalos o extremos y ver la necesidad de analizarlos para su inclusión en el estudio o bien descartalos.

\footnotetext{
${ }^{1}$ Estos casos forman parte de los reportes internos de cátedras como Investigación Operativa y Cuadro de Mando Integral (Facultad de Cs. Exactas, UNCPBA) desarrollados durante los ciclos 2010-2014 y recorren una amplia gama de temas como lo son la aplicación en el contexto de la salud (prestaciones médicas, farmacia), la gestión de stocks, el ámbito académico, las finanzas.
} 
- Tipo de indicador: este dato puede acotar los métodos a utilizar, por ejemplo a la hora de clasificar un conjunto de valores históricos del indicador.

- Estado del indicador: el estado del indicador no es un dato con el que se cuenta al inicio de un proyecto, excepto para el valor actual, por lo que será necesario encontrar al menos una primera instancia de solución a esta problemática para poder utilizar, por ejemplo, métodos de pronósticos sobre esta propiedad.

Respecto del software que utilizamos inicialmente para nuestros ensayos, seleccionamos software libre (SCILAB e INFOSTAT) y también plantillas desarrolladas en planillas de cálculo. Posteriormente y debido a que estas herramientas no interactúan con nuestras $\mathrm{BD}$, desarrollamos prototipos que a su vez nos permitieron agilizar la tarea.

Mencionamos antes que elegimos dos propiedades de los indicadores. Por un lado, para realizar el análisis tradicional sobre el valor elegimos los métodos de Mínimos Cuadrados y Suavizado Exponencial tal como se desarrollan en ITESM (2006).

Es importante señalar que este trabajo abarca el caso particular de regresión lineal de mínimos cuadrados como un primer paso para el análisis del comportamiento de otras funciones (polinómicas, exponencial, etc.).

Para pronosticar por sobre el estado del indicador, elegimos dos métodos que nos dan probabilidades de ocurrencia de los estados, para así poder conocer en qué estado se encontrará un indicador eligiendo la mayor. Los métodos elegidos son Bayes y Markov según conceptos emitidos por Arapé (2000) y Rojo (et al. 2009) respectivamente.

Para la aplicación del método de Bayes, es necesario contar con las probabilidades de cada hipótesis. Dado que en este caso de estudio se eligió el tipo de indicador semáforo, se debe contar con la probabilidad de que el estado del indicador sea CRÍTICO, ACEPTABLE u ÓPTIMO (rojo, amarillo o verde).

Similarmente a lo ocurrido con la aplicación del método de Bayes, debido a la elección del tipo semáforo (tres estados), hemos decidido como más apropiada la utilización de los cuartiles para la clasificación de los mismos, pudiendo re-agruparse los cuatro grupos en tres partes.

Respecto de los ensayos, algunos de estos métodos requieren de una intervención mínima de un usuario, que llamamos opiniones. Por ejemplo, para el método de suavizado exponencial, realizamos ensayos con dos valores del coeficiente alfa (0.15 y 0.75). La elección de la constante de suavizado es crucial en la estimación de pronósticos futuros. Si la serie de tiempo contiene una variabilidad aleatoria sustancial, se preferirá un valor pequeño como constante de suavizado. Por el contrario, para una serie de tiempo con una variabilidad aleatoria relativamente pequeña, valores más elevados de la constante de suavizado tienen la ventaja de ajustar con rapidez los pronósticos. Para el método de Bayes con dos conjuntos de probabilidades <0.5, 0.3, 0.2> y <0.2, 0.4, 0.4> respectivamente.

Los métodos de clasificación nos permiten aproximar los estados de los indicadores en los períodos anteriores, dado que no contamos con esta información al inicio del proyecto. Para clasificar utilizamos el método de cuantiles basados en la conclusiones de Berenson (et al. 1996).

Por otro lado fue necesario diseñar algunos escenarios posibles de aplicación para conformar los procedimientos. 


\subsection{Escenarios de comprobación}

Los métodos de pronósticos fueron probados en distintos escenarios de manera que, utilizando un subconjunto de datos para la obtención de resultados, se puedan comparar los resultados de los pronósticos con datos históricos del indicador.

A. Primer escenario: todos los datos disponibles como subconjunto de datos de entrada para pronosticar los próximos n valores.

B. Segundo escenario: los datos del último período como subconjunto de datos de entrada para pronosticar los próximos n valores.

En ambos escenarios hemos elegido arbitrariamente pronosticar los próximos tres valores del siguiente período, pudiendo el decisor elegir la cantidad de valores que desee pronosticar acorde a sus necesidades.

En la tabla 2 se puede observar un ejemplo de la elección de un segundo escenario. El período seleccionado para el subconjunto de datos de entrada es desde el 01/01/2011 hasta el 01/12/2011 para pronosticar mensualmente en 2012. Los subconjuntos de datos de entrada para pronosticar y para verificar el pronóstico obtenido:

Tabla 2 - Subconjunto de datos del indicador "Crédito total en monto" para el segundo escenario.

\begin{tabular}{|c|c|c|c|}
\hline Fecha & Valor & Estado & Subconjuntos de datos \\
\hline 01/08/2007 & 576632,41 & ACEPTABLE & \\
\hline$\ldots$ & $\cdots$ & $\cdots$ & \\
\hline 01/05/2010 & 348582,04 & ÓPTIMO & \\
\hline$\ldots$ & $\cdots$ & $\cdots$ & \\
\hline 01/01/2011 & 813192,71 & ACEPTABLE & \multirow{4}{*}{$\begin{array}{l}\text { Subconjunto de datos de entrada } \\
\text { para pronosticar }\end{array}$} \\
\hline$\cdots$ & $\cdots$ & $\cdots$ & \\
\hline $01 / 10 / 2011$ & 1028772,93 & CRÍTICO & \\
\hline $01 / 11 / 2011$ & 963597,98 & CRÍTICO & \\
\hline $01 / 12 / 2011$ & 925276,60 & CRÍTICO & \multirow{5}{*}{$\begin{array}{l}\text { Subconjunto de datos para } \\
\text { verificar el pronóstico obtenido }\end{array}$} \\
\hline $01 / 01 / 2012$ & 909961,33 & CRÍTICO & \\
\hline 01/02/2012 & 896456,38 & ACEPTABLE & \\
\hline$\ldots$ & $\ldots$ & $\ldots$ & \\
\hline 01/07/2012 & 904578,51 & CRÍTICO & \\
\hline
\end{tabular}




\subsection{Configuración general para todos los métodos}

El indicador seleccionado en este caso es "Crédito total en monto". Este indicador es de tipo mensual por lo que los siguientes períodos, según el subconjunto para pronosticar, corresponden al primer, segundo y tercer mes del año siguiente (01/01/2012, 01/02/2012, 01/03/2012). Los resultados de los pronósticos se comparan en las mismas fechas. Todos los valores de los indicadores están expresados en pesos (\$).

Los rangos de aceptación, según la clasificación por el método de cuantiles resultaron:

$\begin{array}{ll}\text { CRÍTICO: } & \text { Mayor o igual a 904578,50 (4to cuartil) } \\ \text { ACEPTABLE: } & \begin{array}{l}\text { Mayor o igual a } 367296,88 \text { y menor que } 904578,50 \\ \text { (2do y 3er cuartil) }\end{array} \\ \text { ÓPTIMO: } & \text { Menor que } 367296,88(1 \mathrm{er} \text { cuartil) }\end{array}$

\subsection{Interpretación de Tablas y resultados}

Para los métodos de Mínimos Cuadrados y de Suavizado Exponencial (Tabla 5), la columna "Estado" dentro del pronóstico es obtenida a partir de la evaluación del valor en los rangos de aceptación definidos precedentemente. Para los métodos de Bayes y Markov (Tabla 6), esta columna resulta de la probabilidad obtenida. Si la dirección del indicador es positiva se asigna el estado "ÓPTIMO" (O) a la probabilidad mayor, luego "ACEPTABLE" (A) y posteriormente "CRÍTICA" (C) a la menor. En caso de que la dirección sea negativa, se procede de forma inversa. La evaluación final se toma del mayor valor de probabilidad obtenido por el pronóstico. Para mostrar la coincidencia del pronóstico con el valor histórico (Tabla 3), dentro de las tablas 5 y 6 se resaltan las celdas en gris.

\subsection{Interpretación gráfica}

Antes de concluir si el método entrega un pronóstico ajustado a la realidad, se realiza el gráfico correspondiente. En este gráfico se representan los datos históricos y los pronosticados según se puede apreciar en cada método. Para evaluar la bondad del método, se comparan los valores histórico y pronosticado en las tablas correspondientes. Por otro lado, el gráfico también muestra en su último tramo, --luego de la línea divisoria entre datos históricos de entrada y valores pronosticados--, cuánto se ajusta el valor pronosticado al valor histórico. Para los métodos de Bayes y Markov no es posible mostrar un gráfico dado que lo que se calcula es el valor del vector de probabilidades.

Se desarrolla a continuación un caso particular a los efectos de mostrar cómo se procede. El método seleccionado para el ejemplo es Suavizado exponencial aplicado al indicador de finanzas "Crédito total en monto" donde la constante de suavizado alfa toma el valor 0.75

Par reflejar el segundo escenario se seleccionan los datos del último período como subconjunto de datos de entrada. Entonces para $\alpha=0,75$, la tabla 3 muestra los siguientes resultados: 
Tabla 3 - Comparación de resultados del segundo escenario de suavizado exponencial con $\alpha=0.75$

\begin{tabular}{lccccl}
\hline & Pronóstico & & \multicolumn{3}{c}{ Históricos } \\
\hline Fecha & Valor & Estado & Fecha & Valor & Estado \\
\hline 01/01/2012 & 939091,92 & CRÍTICO & 01/01/2012 & 909961,33 & CRÍTICO \\
\hline 01/02/2012 & 917243,98 & CRÍTICO & 01/02/2012 & 896456,38 & ACEPTABLE \\
\hline 01/03/2012 & 901653,28 & ACEPTABLE & $01 / 03 / 2012$ & 901764,27 & ACEPTABLE \\
\hline
\end{tabular}

La figura 1 muestra gráficamente los datos del segundo escenario donde se pueden observar el tanto los datos de entrada para pronosticar, como así también los utilizados para verificar el pronóstico.

El resultado arrojado es que el método aproxima correctamente en el primer y tercer período de pronóstico.

Repitiendo el procedimiento con los otros métodos seleccionados se arriba a los resultados comparables en las tablas 5 y 6 y en las figuras 2 y 3 .

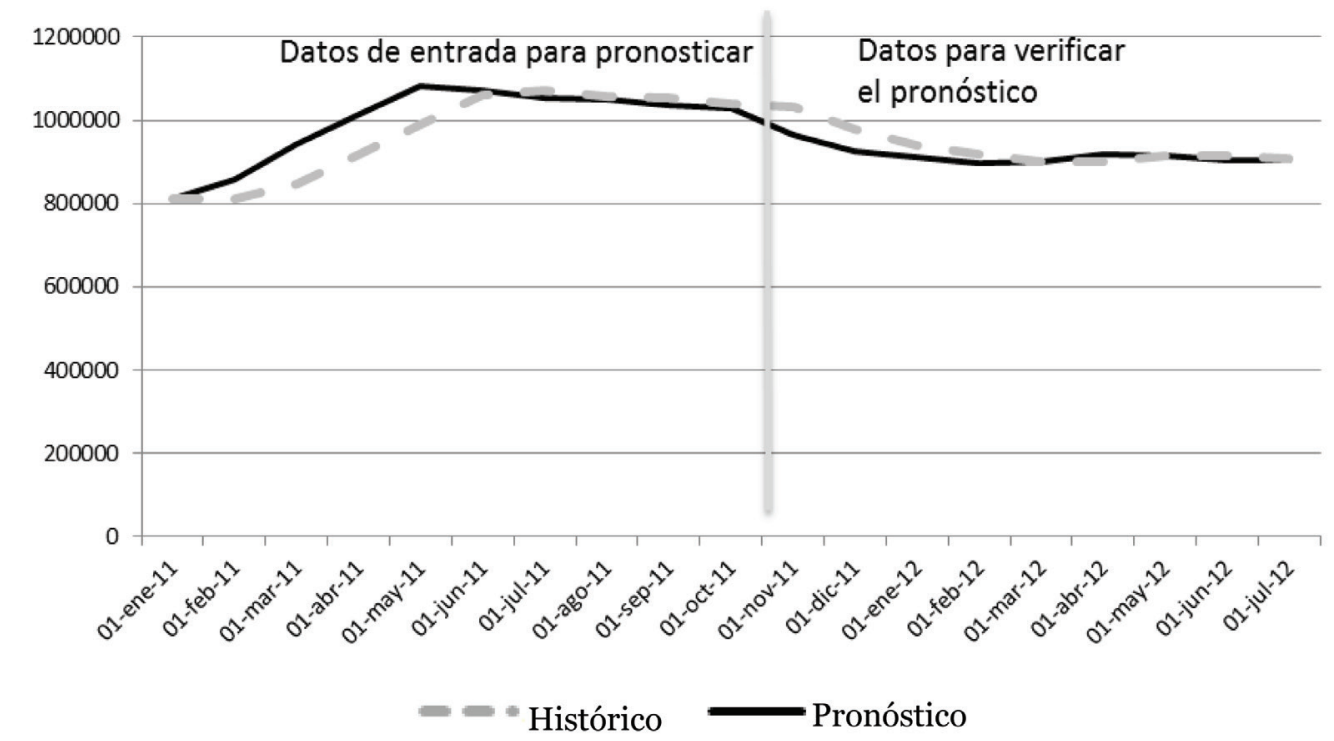

Figura 1 - Comparación de resultados del segundo escenario de suavizado exponencial con $\alpha=0.75$ 
Tabla 4 - Valores históricos de los indicadores utilizados para la comprobación de resultados. Indicador "Crédito total en monto", caso de estudio "Finanzas".

\begin{tabular}{lll}
\hline Fecha & Valor & Estado \\
\hline $01 / 01 / 2012$ & 909961,33 & CRITICO (C) \\
\hline $01 / 02 / 2012$ & 896456,38 & ACEPTABLE (A) \\
\hline $01 / 03 / 2012$ & 901764,27 & ACEPTABLE (A) \\
\hline
\end{tabular}

Tabla 5 - Comparación de resultados de pronósticos sobre el valor del indicador utilizando la mayor cantidad de datos disponibles (primer escenario). Indicador "Crédito total en monto", caso de estudio "Finanzas".

\begin{tabular}{llllllllll}
\hline Mínimos cuadrados & \multicolumn{7}{c}{ Suavizado exponencial a= o,15 } & \multicolumn{2}{c}{ Suavizado exponencial a= o,75 } \\
Fecha & Valor & Estado & Fecha & Valor & Estado & Fecha & Valor & Estado \\
\hline $01 / 01 / 2012$ & 765023,10 & $\mathrm{~A}$ & $01 / 01 / 2012$ & 928304,94 & $\mathrm{C}$ & $01 / 01 / 2012$ & 939092,00 & $\mathrm{C}$ \\
\hline $01 / 02 / 2012$ & 770404,60 & $\mathrm{~A}$ & $01 / 02 / 2012$ & 928385,10 & $\mathrm{C}$ & $01 / 02 / 2012$ & 970176,40 & $\mathrm{C}$ \\
\hline $01 / 03 / 2012$ & 775786,10 & $\mathrm{~A}$ & $01 / 03 / 2012$ & 928373,10 & $\mathrm{C}$ & $01 / 03 / 2012$ & 901653,28 & $\mathrm{~A}$ \\
\hline
\end{tabular}

Tabla 6 - Comparación de resultados de pronósticos sobre el estado del indicador utilizando la mayor cantidad de datos disponibles (primer escenario). Indicador "Crédito total en monto", caso de estudio "Finanzas".

\begin{tabular}{|c|c|c|c|c|c|c|c|c|c|c|c|}
\hline \multicolumn{4}{|c|}{ Bayes 1ra opinión } & \multicolumn{5}{|c|}{ Bayes 2da opinión } & \multicolumn{3}{|c|}{ Markov } \\
\hline \multirow[t]{2}{*}{ Fecha } & \multicolumn{2}{|r|}{ Valor } & \multirow{4}{*}{$\begin{array}{c}\text { Estado } \\
\text { A }\end{array}$} & \multirow{4}{*}{$\begin{array}{l}\text { Fecha } \\
\text { 01/o1 } \\
\text { /2012 }\end{array}$} & \multicolumn{2}{|c|}{ Valor } & \multirow{4}{*}{$\begin{array}{c}\text { Estado } \\
\text { A }\end{array}$} & \multirow{4}{*}{$\begin{array}{l}\text { Fecha } \\
\text { 01/o1 } \\
\text { /2012 }\end{array}$} & \multicolumn{2}{|c|}{ Valor } & \multirow{4}{*}{$\begin{array}{c}\text { Estado } \\
\text { O }\end{array}$} \\
\hline & $\mathrm{P}(\mathrm{C})$ & $4,11 \mathrm{E}-12$ & & & $\mathrm{P}(\mathrm{C})$ & $1,42 \mathrm{E}-\mathrm{O} 4$ & & & $\mathrm{P}(\mathrm{C})$ & 0,00 & \\
\hline \multirow{2}{*}{$\begin{array}{l}\text { O1/O1 } \\
/ 2012\end{array}$} & $\mathrm{P}(\mathrm{A})$ & 1,00 & & & $\mathrm{P}(\mathrm{A})$ & 0,99 & & & $\mathrm{P}(\mathrm{A})$ & 0,48272 & \\
\hline & $\mathrm{P}(\mathrm{O})$ & $5,47 \mathrm{E}-18$ & & & $\mathrm{P}(\mathrm{O})$ & $6,20 \mathrm{E}-\mathrm{O} 1$ & & & $\mathrm{P}(\mathrm{O})$ & 0,51727 & \\
\hline \multirow{3}{*}{$\begin{array}{l}\text { O1/o2 } \\
/ 2012\end{array}$} & $\mathrm{P}(\mathrm{C})$ & $1,59 \mathrm{E}-12$ & \multirow{3}{*}{ A } & \multirow{3}{*}{$\begin{array}{l}\text { 01/O2 } \\
\text { /2012 }\end{array}$} & $\mathrm{P}(\mathrm{C})$ & $6,18 \mathrm{E}-\mathrm{O} 4$ & \multirow{3}{*}{ A } & \multirow{3}{*}{$\begin{array}{l}\text { 01/o2 } \\
/ 2012\end{array}$} & $\mathrm{P}(\mathrm{C})$ & 0,18515 & \\
\hline & $\mathrm{P}(\mathrm{A})$ & 1,00 & & & $\mathrm{P}(\mathrm{A})$ & 0,99 & & & $\mathrm{P}(\mathrm{A})$ & 0,51864 & A \\
\hline & $\mathrm{P}(\mathrm{O})$ & $3,01 \mathrm{E}-17$ & & & $\mathrm{P}(\mathrm{O})$ & $4,98 \mathrm{E}-02$ & & & $\mathrm{P}(\mathrm{O})$ & 0,2962 & \\
\hline \multirow{3}{*}{$\begin{array}{l}\text { O1/O3 } \\
/ 2012\end{array}$} & $\mathrm{P}(\mathrm{C})$ & $1,18 \mathrm{E}-13$ & \multirow{3}{*}{ A } & \multirow{3}{*}{$\begin{array}{l}\text { 01/o3 } \\
/ 2012\end{array}$} & $\mathrm{P}(\mathrm{C})$ & $3,85 \mathrm{E}-\mathrm{O} 5$ & \multirow{3}{*}{ A } & \multirow{3}{*}{$\begin{array}{l}\text { 01/o3 } \\
\text { /2012 }\end{array}$} & $\mathrm{P}(\mathrm{C})$ & 0,18535 & \\
\hline & $\mathrm{P}(\mathrm{A})$ & 1,00 & & & $\mathrm{P}(\mathrm{A})$ & 0,99 & & & $P(A)$ & 0,51809 & A \\
\hline & $\mathrm{P}(\mathrm{O})$ & $2,23 \mathrm{E}-18$ & & & $\mathrm{P}(\mathrm{O})$ & $5,75 \mathrm{E}-\mathrm{O} 2$ & & & $\mathrm{P}(\mathrm{O})$ & 0,29655 & \\
\hline
\end{tabular}


Mínimos cuadrados

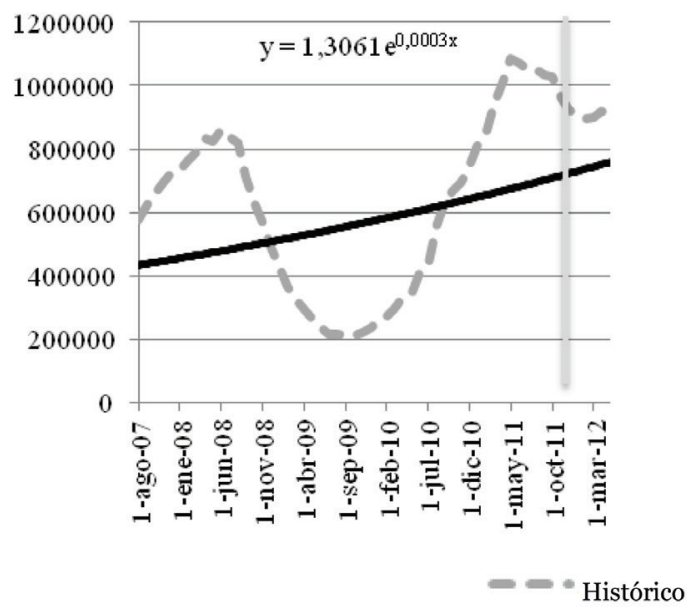

Suavizado exponencial $(\alpha=0.15)$

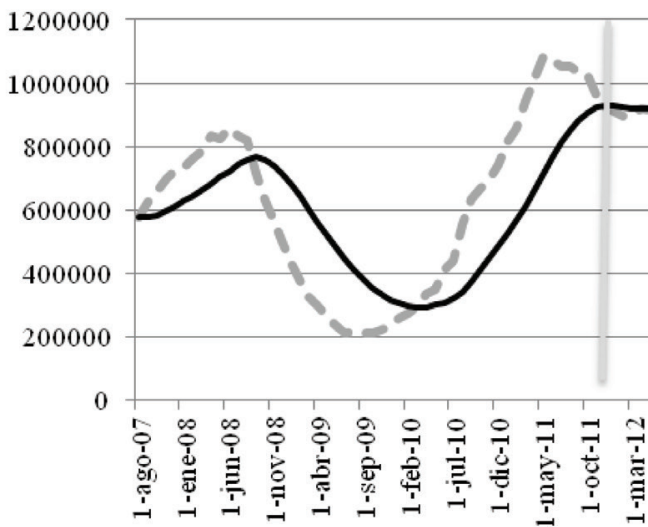

Pronóstico

Figura 2 - Comprobación de los métodos mediante representación gráfica, utilizando la mayor cantidad de datos disponibles (primer escenario). Indicador "Crédito total en monto", caso de estudio "Finanzas".

Mínimos cuadrados

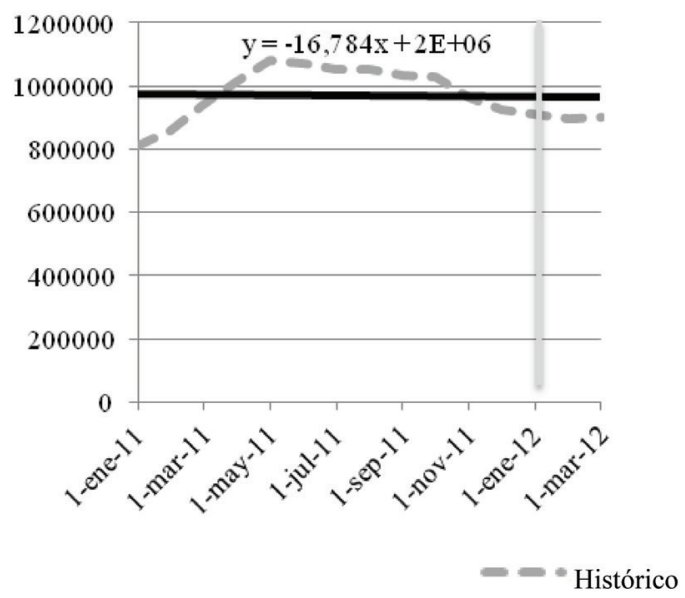

Suavizado exponencial $(\alpha=0.15)$

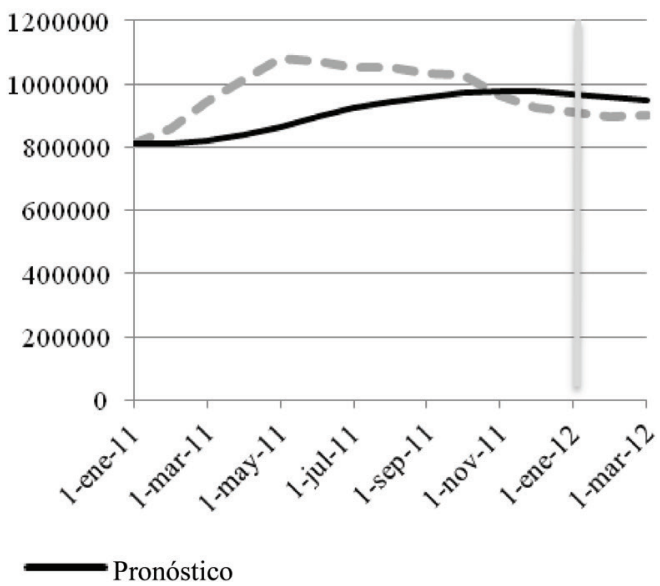

Figura 3 - Comprobación de los métodos mediante representación gráfica utilizando los datos del último período (segundo escenario). Indicador "Crédito total en monto", caso de estudio "Finanzas". 


\section{Conclusiones}

En la actualidad los proyectos de sistemas de indicadores se centran en encontrar el conjunto apropiado deindicadores para gestionar la organización aplicando metodologías como por ejemplo el CMI, perdiendo de vista las múltiples facetas de lo que implica un indicador en sí mismo. La información que nos aporta un indicador existía antes de que éste fuera definido por lo que resulta imprescindible contar con su aporte. Por este motivo nuestra propuesta se orienta a trabajar con métodos matemáticos que analicen la historia de los datos para proveer mayor información a la organización antes que solo contar con una medida del valor actual.

Por otra parte la selección de indicadores implica la necesidad de definir parámetros o umbrales para poder evaluar la evolución del cumplimiento de los mismos. Dada la marcada dependencia creada por las organizaciones respecto de personas expertas que definen esta situación, torna la base de este análisis de carácter práctico pero subjetivo y no adaptable a las condiciones cambiantes del entorno operativo. Su efectividad depende fuertemente de la experiencia y sesgo personal de quien lo define y por lo tanto no posee rigor científico.

\subsection{Conclusiones del caso de estudio}

Se puede apreciar que el pronóstico del primer período, en este caso el primer mes, influencia el pronóstico de los períodos subsiguientes, lo cual se refleja en los valores sucesivos independientemente del método utilizado.

Si bien los métodos utilizados para el pronóstico sobre el valor del indicador muestran similar ajuste, cabe notar una diferencia. El método de mínimos cuadrados extrapola un valor pronóstico para el primer período que permite asignar el estado pronosticado. Dependiendo de la pendiente de la regresión lineal, el estado pronosticado puede ajustarse correctamente a la realidad o no debido a la discretización de los estados. Por otro lado, el método de suavizado exponencial lo hace directamente sobre el valor, lo cual parece brindar una aproximación más apropiada.

Notar que el método de mínimos cuadrados ajusta los resultados recién en el segundo período (Tabla 5), lo cual lo hace menos confiable de acuerdo con lo mencionado en primer lugar.

En función a la observación de los resultados obtenidos luego de aplicar los métodos para pronosticar el estado del indicador, podemos ver claramente que las mejores aproximaciones se logran en el primer escenario, esto es, cuando se utiliza una mayor cantidad de datos históricos para pronosticar. En este escenario tanto el método de Bayes como el de Markov coinciden en las aproximaciones.

\subsection{Conclusiones generales}

La metodología fue aplicada a otros casos de estudio de diferente contexto (configurandola BD de prestaciones médicas, farmacia, stocks de repuestos, registro de datos académicos), utilizando los mismos protocolos. En todos los casos se observaron resultados similares respecto de la bondad de cada uno de los cuatro métodos matemáticos, notando las diferencias en las respuestas en función de los contextos de cada caso. Esto permite considerar el procedimiento aquí propuesto como una metodología válida y confiable. 


\section{Referencias}

Arapé J. (2000). Manual de Metodologías Tomo II. La Técnica Bayesiana. Secretaría de las Naciones Unidas para el Desarrollo Industrial (ONUDI). Programa de Prospectiva Tecnológica para Latinoamérica y el Caribe. Editado por Visión Grupo Consultores de Venezuela. Disponible en: www.cgee.org.br/atividades/redirKori/848

Berenson M., Levine D. (1996). Estadística Básica en Administración, Conceptos y Aplicaciones. Sexta edición. Prentice Hall Hispanoamericana, S. A. México.

Blumenberg S., Hinz D., Goethe J. (2006). Enhancing the Prognostic Power of it Balanced Scorecards with Bayesian Belief Networks. University, Frankfurt, Germany. Proceedings of the 39th Hawaii International Conference on System Sciences. 0-7695-2507-5/06 (c) 2006 IEEE.

Chan S., Ip W. (2010). A Scorecard-Markov Model for New Product Screening Decisions. Department of Industrial and Systems Engineering, The Hong Kong Polytechnic University. Industrial Management \& Data Systems Vol. 110 No. 7, pp. 971-992. (c) Emerald Group Publishing Limited.

Illescas G., Sanchez-Segura M., Xodo D. (2014). Una aproximación a la aplicación de métodos matemáticos en el control de gestión por indicadores. Revista EPIO $\mathrm{N}^{\circ} 35$, año XXII (Índice: LATINDEX), Argentina. pp 199-215.

ITESM (2006). Métodos Estadísticos para la Estimación de Ingresos. Instituto Tecnológico y de Estudios Superiores de Monterrey, México. Diplomado en Gestión Estratégica de las Finanzas Públicas. Disponible en: http://www.cca.org.mx/ funcionarios/biblioteca/html/finanzas_publicas/documentos/3/m3_metodos.pdf

Kaplan R., Norton D. (1996). Using the Balanced Scorecard as a Strategic Management System. Harvard Business Review, enero-febrero.

Köppen V., Allgeier M., Lenz H. (2007). Balanced Scorecard Simulator - A Tool for Stochastic Business Figures. Studies in Classification, Data Analysis, and Knowledge Organization. Advances in Data Analysis, Part VI, Pages 457-464. Institute of Information Systems, Free University Berlin, D-14195 Berlin, Germany.

Rojo H., Miranda M. (2009). Cadenas de Markov. Cátedra de Investigación Operativa Facultad de Ingeniería, Universidad de Buenos Aires. Disponible en: http:// operativa7107.awardspace.com/apuntes/MarkovParte1.pdf 